\title{
Autofocus for digital Fresnel holograms by use of a Fresnelet-sparsity criterion
}

\author{
Michael Liebling \\ Biological Imaging Center, California Institute of Technology, Mail Code 139-74, Pasadena, California 91125 \\ Michael Unser \\ Biomedical Imaging Group, École Polytechnique Fédérale de Lausanne, Bâtiment de Microtechnique 4.127, \\ CH-1015 Lausanne, Switzerland
}

Received January 23, 2004; revised manuscript received June 7, 2004; accepted July 19, 2004

\begin{abstract}
We propose a robust autofocus method for reconstructing digital Fresnel holograms. The numerical reconstruction involves simulating the propagation of a complex wave front to the appropriate distance. Since the latter value is difficult to determine manually, it is desirable to rely on an automatic procedure for finding the optimal distance to achieve high-quality reconstructions. Our algorithm maximizes a sharpness metric related to the sparsity of the signal's expansion in distance-dependent waveletlike Fresnelet bases. We show results from simulations and experimental situations that confirm its applicability. (C) 2004 Optical Society of America

OCIS codes: $090.0090,100.3010,100.7410,110.3000,090.1000$.
\end{abstract}

\section{INTRODUCTION}

The computerized reconstruction of complex-valued object waves from Fresnel holograms ${ }^{1}$ acquired electronically and digitized ${ }^{2-8}$ relies on the numerical computation of wave propagation. A possible approach for a wide variety of setups is to consider the free-space propagation formula in the Fresnel approximation, which relates the complex values of a propagating complex scalar wave measured in two planes perpendicular to the direction of propagation and separated by a distance $d$ (see Fig. 1). The Fresnel approximation is defined ${ }^{9}$ as

$$
\begin{aligned}
f_{d}(\mathbf{x}) & =\mathcal{R}_{d}\{f\}(\mathbf{x}) \\
& =\frac{\exp \left(i k_{\lambda} d\right)}{i \lambda d} \iint f\left(\mathbf{x}^{\prime}\right) \exp \left(\frac{i \pi}{\lambda d}\left\|\mathbf{x}-\mathbf{x}^{\prime}\right\|^{2}\right) \mathrm{d} \mathbf{x}^{\prime},
\end{aligned}
$$

where $\lambda$ is the wavelength of the light and $k_{\lambda}=2 \pi / \lambda$ its wave number, and $\mathbf{x}=(x, y)$. In a holography experiment, the interference of the diffracted wave with a reference wave is recorded on a CCD and can be written as

$$
I(\mathbf{x})=\left|f_{d}(\mathbf{x})+R(\mathbf{x})\right|^{2} .
$$

The reconstruction of the complex-valued wave $f$ in the object's vicinity from one or several measurements of $I$ may be accomplished conveniently in two steps: First, one reconstructs the wave $f_{d}$ in the CCD plane from $I$, for example by use of a parametric phase-retrieval procedure, ${ }^{10}$ and second, one computes $f$ through an appropriate discretization of Eq. (1). To perform the latter operation, the distance parameter $d$ in Eq. (1) must be set accurately. We have proposed elsewhere ${ }^{11}$ a numerical, multiresolution, reconstruction implementation for evaluating Eq. (1) based on a Fresnelet decomposition. Here we show how this procedure can also be used advantageously to adjust the focusing parameter $d$ in an accurate, robust, and fast manner. Our method is based on the maximization of the sparsity of the Fresnelet representation, which appears to be a natural choice in light of the multiresolution, wavelet-transformlike reconstruction method we have adopted.

The paper is organized as follows. In Section 2 we list a number of possible approaches to autofocusing. In Section 3 we emphasize the concept of sparse image representations, which is central to our approach. In Section 4 we give the formal definition of distance-dependent Fresnelet bases and of the Fresnelet-based propagation algorithm. In Section 5 we introduce the autofocus algorithm and its underlying sparsity measure. In Section 6 we illustrate and validate the method using both synthetic (simulated) and true measurement data.

\section{EXISTING METHODS}

A. Image Quality Functionals

Although our algorithm is specifically designed for the reconstruction of complex Fresnel fields, it is in keeping with applications that aim at synthesizing and acquiring images as well as assessing their quality through the evaluation (and eventually the determination of an extremum) of an image quality functional that depends on some imaging parameter to be optimized-here, the distance. More specifically, such procedures are used for providing feedback to acquisition devices for automatically setting the distance and alignment between their constituents as in optical imaging, ${ }^{12,13}$ electronic microscopy, ${ }^{14}$ and holography ${ }^{15}$ or for the optical reconstruction of acoustic holograms. ${ }^{16}$ They also appear in devices that actively correct the incoming wave front to 


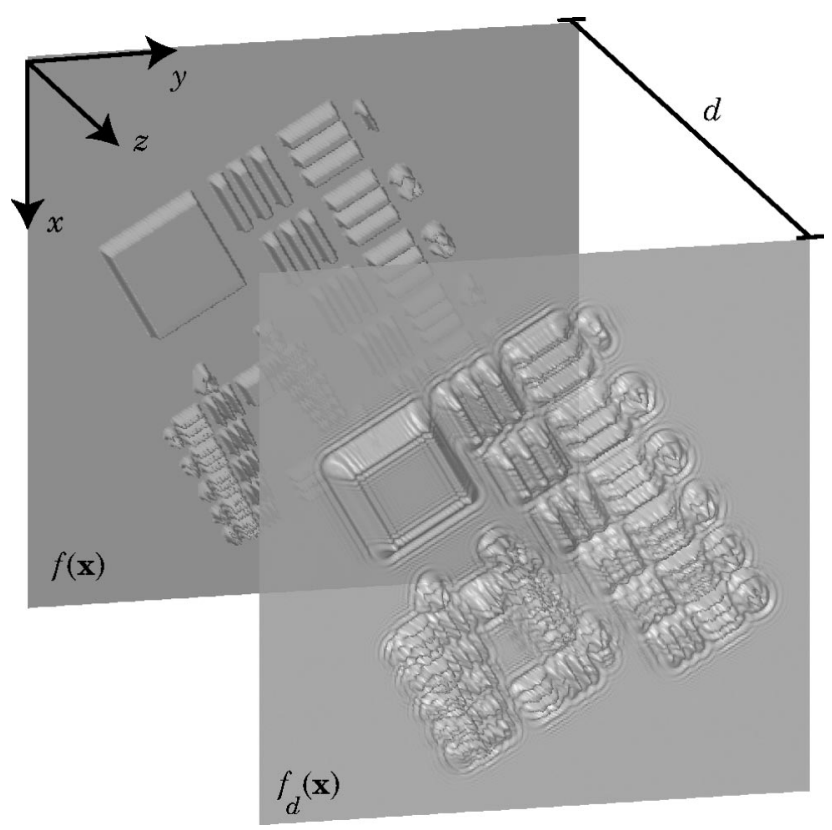

Fig. 1. Fresnel propagation.

overcome aberrations in astronomical imaging ${ }^{17,18}$ or microscopy. ${ }^{19}$ Yet another use of such functionals is image quality assessment of acquisition devices and displays. $^{20}$ They are central for local sharpness evaluation in the case of image fusion and depth evaluation in images of three-dimensional scenes ${ }^{21,22}$ as well as for determining particle location. They are also instrumental in the estimation of unknown imaging parameters for degraded image restoration, ${ }^{23}$ digital aberration correction and deconvolution, and computerized image reconstruction for various modalities, including coherent imaging. ${ }^{24,25}$ The automatic setting of the distance parameter for numerical reconstruction of complex wave fields from digitally acquired holograms (digital holography) also falls into this last category. ${ }^{26}$

The selection of a suitable image quality metric is usually an ad hoc choice driven by the imaging system's characteristics. Insensitivity to specific conditions and invariance with respect to diverse transforms ${ }^{27,28}$ constitute other goals. The perfect functional should be a unimodal function over a wide range of parameter values with a low computational cost. ${ }^{29}$ Also, defining sharpness in the first place implies agreement on some a priori knowledge of the image to reconstruct. Possible requirements might be to achieve images with high contrast, sharp edges, or crisp details. Such criteria are highly application dependent and possibly difficult to apply in the presence of noise.

\section{B. Related Work}

Gillespie and $\mathrm{King}^{26}$ have proposed to use the reconstructed image's self-entropy for measuring the focus, albeit with quantized levels of gray. Ferraro et al. ${ }^{30}$ have recently proposed an autofocus algorithm for digital inline holography that tracks the axial displacement of the sample in real time by measuring the phase shift of the hologram fringes. The use of an autofocus method has also been reported by Hobson and Watson, ${ }^{31}$ although they do not give details of its implementation.

We are aware of at least three uses of wavelets for focus estimation and-or holographic reconstruction, albeit unrelated to the method we propose. Widjaja and Jutamulia $^{32}$ proposed to improve a focus measure based on the autocorrelation of an image whose edges have been enhanced through a continuous wavelet transform. Rooms et $a l .{ }^{33}$ estimated image blur by analyzing the sharpness of its sharpest edges through evaluation of the Lipschitz exponent based on the analysis of the scalogram obtained by continuous wavelet transform. Onural and Kocatepe $^{34}$ proposed methods for hologram reconstruction and space-depth analysis for the three-dimensional determination of particle location by use of scaling-chirp functions. Their formulation of the Fresnel diffraction formula makes it isomorphic with the continuous wavelettransform formulation, provided the commonly used admissibility condition is extended appropriately.

\section{SPARSE IMAGE REPRESENTATIONS}

A standard approach in image processing and optics is to express the signal of interest as a weighted sum of basis functions. Fourier, Hermite-Gauss, or wavelet bases are among the most popular candidates for such expansions. Although it is possible in theory to express a finite energy signal in any of these bases, in practice the choice is usually dictated by specific properties of the basis functions. A family might be selected, for example, because it diagonalizes an operator that is relevant to the application at hand (e.g., the Hermite-Gauss modes for fiber-optics problems). Another reason for choosing a specific basis is that it may yield a sparse representation of the signal; i.e., most of the signal's energy is packed into a few coefficients only. Local Gabor representations, and in particular wavelet bases, ${ }^{35}$ are good candidates to yield sparse representations of natural images. ${ }^{36,37}$ The reason for the wavelet transform's excellent energy compaction properties for a large palette of images is that wavelets are well localized and they yield very small coefficients in smooth signal regions, thanks to their vanishing-moments properties. This energy-compaction property of the wavelet transform was recognized early on and has proved to be useful in a wide variety of applications, ranging from superresolution image restoration, ${ }^{38}$ efficient noise-reduction algorithms, ${ }^{39,40}$ and state-of-theart image compression algorithms, ${ }^{41}$ including the recently adopted JPEG 2000 standard. $^{42}$ Sparse representations also play an important role in recently proposed blind-source-separation algorithms. ${ }^{43}$

Fresnel fields and holograms are not natural images, and their decomposition is sparse only if the basis functions are well chosen. Our guiding principle for reconstructing focused wave fronts is that their expansion in a distance-dependent basis should be sparse.

\section{FRESNELETS}

\section{A. Definition}

We consider the separable orthonormal wavelet basis of $L_{2}\left(\mathbf{R}^{2}\right)$ (Ref. 44, pp. 304-306): 


$$
\left\{\psi_{j, \mathbf{m}}^{1}(\mathbf{x}), \psi_{j, \mathbf{m}}^{2}(\mathbf{x}), \psi_{j, \mathbf{m}}^{3}(\mathbf{x})\right\}_{j \in \mathbf{Z}, m \in \mathbf{Z}^{2}},
$$

where the two-dimensional wavelets

$$
\psi_{j, \mathbf{m}}^{p}(\mathbf{x})=\frac{1}{2^{j}} \psi^{p}\left(\frac{\mathbf{x}}{2^{j}}-\mathbf{m}\right)
$$

are constructed with separable products of the cubic Battle-Lemarié scaling function $\phi(x)$ and wavelet $\psi(x)$ (Refs. 45 and 46):

$$
\begin{gathered}
\psi^{1}(\mathbf{x})=\phi(x) \psi(y), \quad \psi^{2}(\mathbf{x})=\psi(x) \phi(y), \\
\psi^{3}(\mathbf{x})=\psi(x) \psi(y) .
\end{gathered}
$$

For the sake of brevity, we index the basis functions with a single index $\mathbf{k}$ that includes the scale $j \in \mathbf{Z}$, translation $m \in \mathbf{Z}^{2}$, and wavelet type $p \in\{1,2,3\}$,

$$
\psi_{\mathbf{k}}(\mathbf{x})=\psi_{j, \mathbf{m}}^{p}(\mathbf{x}), \quad \mathbf{k}=(p, j, \mathbf{m}),
$$

and denote the wavelet basis in Eq. (3) by $\left\{\psi_{\mathbf{k}}\right\}_{\mathbf{k}}$. In practice one considers only a finite number of scales and translates (because of the finite resolution and support of the image, and appropriate boundary conditions).

A complex wave field $f(\mathbf{x})$, measured in a plane perpendicular to propagation, may be decomposed in this basis according to

$$
f(\mathbf{x})=\sum_{\mathbf{k}} c_{\mathbf{k}} \psi_{\mathbf{k}}(\mathbf{x})
$$

where the coefficients $c_{\mathbf{k}}$ are given by the inner products

$$
\begin{aligned}
c_{\mathbf{k}} & =\left\langle f, \psi_{\mathbf{k}}\right\rangle \\
& =\int_{-\infty}^{\infty} f^{*}(\mathbf{x}) \psi_{\mathbf{k}}(\mathbf{x}) \mathrm{d} \mathbf{x} .
\end{aligned}
$$

Note that we consider integer scale and shift parameters $j$ and $\mathbf{m}$, which leads to a dyadic multiresolution structure. ${ }^{35}$ This approach is different from the representation provided by continuous wavelet transforms, which is highly redundant (i.e., not sparse) and does not have an underlying basis.

For any distance $d \neq 0$, an associated Fresnelet basis $\left\{\psi_{\mathbf{k}}^{d}(\mathbf{x})\right\}_{\mathbf{k}}$ of $L_{2}\left(\mathbf{R}^{2}\right)$ can be constructed by taking the Fresnel transform of the basis $\left\{\psi_{\mathbf{k}}\right\}_{\mathbf{k}}$, viz.,

$$
\psi_{\mathbf{k}}^{d}(\mathbf{x})=\mathcal{R}_{d}\left\{\psi_{\mathbf{k}}\right\}(\mathbf{x}) .
$$

The transformed basis functions $\psi_{\mathbf{k}}^{d}$ are shift invariant on a level-by-level basis, but their multiresolution properties are governed by the special form that the dilation operator takes in the Fresnel domain. ${ }^{11}$ Such bases have many desirable properties required for the digital processing of holograms: For example, since they are related to splines, whose Fresnel transform may be computed analytically, ${ }^{11}$ they are well defined in both time and frequency. Here we will show that they become particularly useful for reconstructing propagated complex wave fields.

\section{B. Fresnelet-Based Propagation}

The propagating wave front may be computed at any depth, given its wavelet coefficients at the origin, by sim- ply replacing the wavelet basis functions in the expansion of Eq. (7) with the Fresnelets associated with a different depth

$$
\begin{aligned}
f_{d}(\mathbf{x}) & =\mathcal{R}_{d}\{f\}(\mathbf{x}) \\
& =\sum_{\mathbf{k}} c_{\mathbf{k}} \psi_{\mathbf{k}}^{d}(\mathbf{x}) .
\end{aligned}
$$

Conversely, the focused wave front $f(\mathbf{x})$ at the origin may be reconstructed, given complex measurements of the propagated field $f_{\bar{d}}(\mathbf{x})$ in a plane at distance $\bar{d}$, as follows:

$$
f(\mathbf{x})=\sum_{\mathbf{k}} c_{\mathbf{k}}^{d} \psi_{\mathbf{k}}(\mathbf{x})
$$

where $c_{\mathbf{k}}^{d}=\left\langle f_{\bar{d}}^{-}, \psi_{\mathbf{k}}^{d}(\mathbf{x})\right\rangle$. It is only when the distance is well adjusted $(d=\bar{d})$ that we have $c_{\mathbf{k}}^{d}=c_{\mathbf{k}}$ and that the reconstruction leads to a focused image. The focus measure that we propose lets us determine the quality of the computed coefficients, that is, the quality of our reconstruction.

\section{SPARSITY OF FRESNELET-TRANSFORM- BASED AUTOFOCUS}

Our starting hypothesis is that the wavelet coefficients $c_{\mathbf{k}}$ for focused images are sparse. To decide whether the computed coefficients $c_{\mathbf{k}}^{d}$ are satisfactory, we need a measure that depends on their sparsity. We define the focus measure $S(d)$ as follows. For a test depth $d$ we compute the Fresnelet coefficients $c_{\mathbf{k}}^{d}=\left\langle f_{\bar{d}}, \psi_{\mathbf{k}}^{d}(\mathbf{x})\right\rangle$. The coefficients are sorted: We define a mapping $\mathbf{k}=\mathbf{k}(l)$ such that

$$
\left|c_{\mathbf{k}(l-1)}^{d}\right| \geqslant\left|c_{\mathbf{k}(l)}^{d}\right| \geqslant\left|c_{\mathbf{k}(l+1)}^{d}\right|,
$$

with $0<l<L$, and where $L$ is the total number of coefficients. The sharpness metric $S(d)$ is the energy of the signal that is reconstructed with the fraction $0<\alpha<1$ of highest modulus coefficients, viz.,

$$
\begin{aligned}
S(d) & =\iint\left|\sum_{l=0}^{\lfloor\alpha L\rfloor} c_{\mathbf{k}(l)}^{d} \psi_{\mathbf{k}(l)}(\mathbf{x})\right|^{2} \mathrm{~d} \mathbf{x} \\
& =\sum_{l=0}^{\lfloor\alpha L\rfloor}\left|c_{\mathbf{k}(l)}^{d}\right|^{2} .
\end{aligned}
$$

The second equality is a consequence of the basis functions $\psi_{\mathbf{k}(l)}$ being orthonormal. In practice, we typically set $\alpha \approx 1 \%$. An initial depth range is defined, for example by rough measurements or estimates made on the experimental setup. For a lensless digital holography setup the distance is usually known within $0.1 \mathrm{~m}$; i.e., the distance range to be considered is approximately $0.2 \mathrm{~m}$. Our autofocus algorithm then maximizes the $S(d)$ criterion. Since computing $S(d)$ is costly, it should be evaluated as few times as possible during maximization. We relied on the MATLAB implementation fminbnd ${ }^{47,48}$ of a derivative-free algorithm due to Brent ${ }^{49}$ that finds local extrema on a bounded interval. At every iteration, depending on the outcome of a suitable test, it selects either a golden section search (that handles the worst possible 
case of function maximization) or a parabola fit through three points that leads to the maximum in a single leap (when the function is parabolic, which is more likely if it is sufficiently smooth, see Ref. 50, pp. 397-405).

The focusing problem may be summarized as follows: We aim to find the best Fresnelet basis such that as much of the image energy is encoded with as few coefficients as possible. This idea is very similar to the following problem of linear algebra in a two-dimensional Cartesian vector space. On the road map shown in Fig. 2, we determine the highway's direction (the reconstruction distance) by using an instrument that is initially oriented toward the North Pole and that measures a test car's two coordinates in an orthonormal basis but displays only the higher (the quality metric). The instrument, hence the basis, is rotated until the device displays the maximum value, which gives us the orientation we are seeking. Some a priori knowledge is, however, required for this method to be effective: The car should be driving on the highway (but not at the origin), and the initial guess should be no more than $45^{\circ}$ away from the true value. It is noteworthy that all the rotated bases, like the Fresnelet bases for different distance parameter $d$, are equivalent in that they all allow expression of the position of any car on the map.

In terms of computational complexity the cost for sorting the $L$ coefficients is $\mathcal{O}(L \log L){ }^{51}$ The cost of computing a Fresnelet transform, which relies on fast Fourier transforms, is of similar complexity, i.e., $\mathcal{O}(L \log L)$. The maximization algorithm usually converges in less than ten iterations. The whole autofocusing procedure for a $512 \times 512$-pixel image takes approximately $9 \mathrm{~s}$ on a PowerPC G5, 1.8-GHz computer, but we believe that the implementation may be optimized for specific applications and to allow performance of real-time processing.

\section{RESULTS AND DISCUSSION}

\section{A. Sparsity Illustration}

We have simulated the propagation of a coherent monochromatic scalar complex wave. The diffracted wave was computed at a distance of $d=0.1 \mathrm{~m}$ by a procedure we

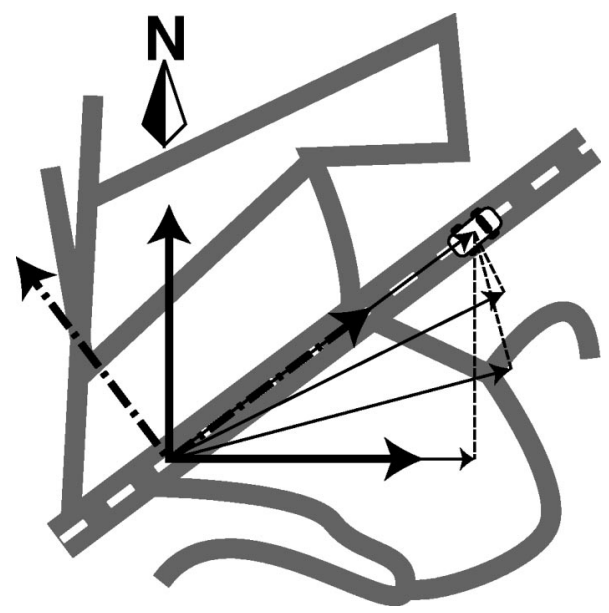

Fig. 2. Orientation of the highway is given by the orientation of the basis in which the representation of a car's position is sparsest.

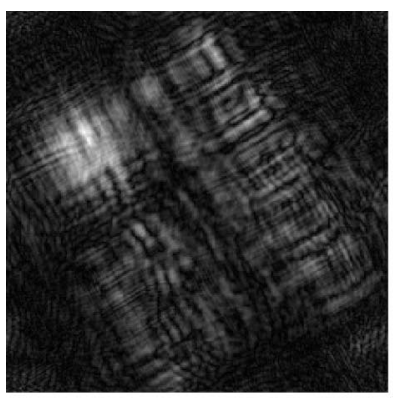

(a)

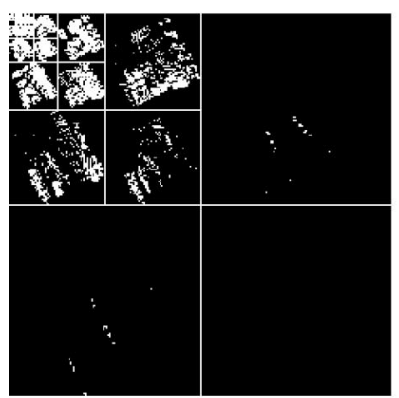

(c)

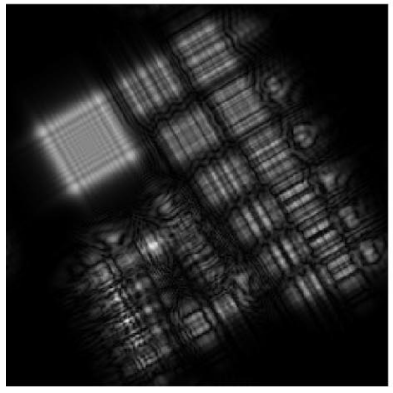

(e)

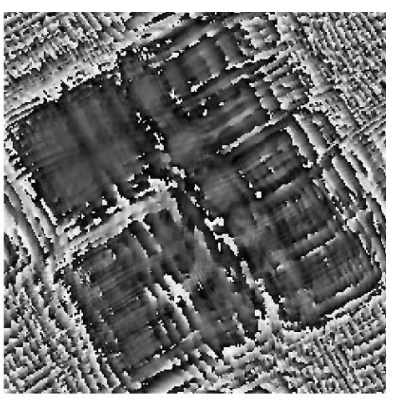

(b)

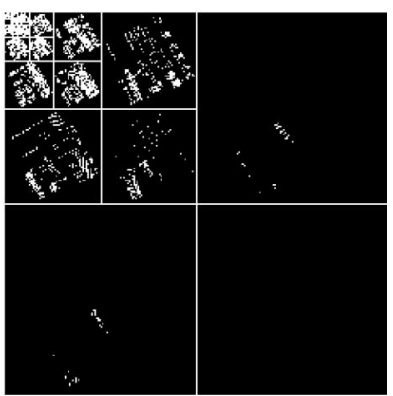

(d)

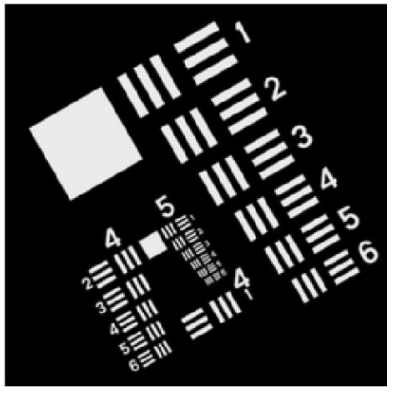

(f)
Fig. 3. Sparsity of wavelet coefficients. (a) Intensity and (b) phase of measured complex field at a distance $d=0.1 \mathrm{~m}$. (c) Positions of the 5\% highest Fresnelet coefficients for parameter $d=0.09 \mathrm{~m}$ that make up $95 \%$ of the signal's energy. (d) The same energy is packed in only $3.1 \%$ of the coefficients when $d$ $=0.1 \mathrm{~m}$. The respective reconstructions are given in (e) and (f).

described elsewhere. ${ }^{11}$ Its intensity and phase are shown in Figs. 3(a) and 3(b). We then applied the Fresnelet decomposition with bases of different distance parameters $d$ ranging from $0.01 \mathrm{~m}$ to $0.19 \mathrm{~m}$. In Figs. $3(\mathrm{c})$ and $3(\mathrm{~d})$ the positions of the wavelet coefficients that account for $95 \%$ of the signal's energy are shown. More than 1.5 times as many coefficients are required when the Fresnelet parameter $d=0.09 \mathrm{~m}$ is used than when the correct parameter $d=0.1 \mathrm{~m}$ is applied. In Fig. 4 we show the Fresnelet coefficient's energy packing for different distances; we have sorted the Fresnelet coefficients in decreasing order and reported their relative cumulated energy. From the inset, it is clearly visible that the larger the distance difference between the Fresnelet parameter and the focus distance, the weaker the energy packing. We show the sharpness metric $S(d)$ in Fig. 5(a) along with two other metrics, the fourth power of the wave's modulus and the squared modulus of the intensity's Laplacian computed as follows: 


$$
\begin{aligned}
& S_{4}(d)=\iint\left|\mathcal{R}_{-d}\left\{f_{\bar{d}}\right\}(\mathbf{x})\right|^{4} \mathrm{~d} \mathbf{x}, \\
& S_{L}(d)=\left.\iint|\Delta| \mathcal{R}_{-d}\left\{f_{\bar{d}}\right\}(\mathbf{x})\right|^{2} \mid \mathrm{d} \mathbf{x} .
\end{aligned}
$$

The maximum is reached for the optimal distance $d$ $=0.1 \mathrm{~m}$ for all three sharpness metrics. However, the Laplacian and squared intensity metrics exhibit local maxima even in our ideal, noiseless situation. In Fig. 5 (b) we show the same curves for the case in which $10 \%$ Gaussian random noise was added to the propagated wave field before reconstruction.

Since Fresnelet coefficients, like wavelet coefficients, may be assimilated to the derivatives of the twodimensional function along the horizontal, vertical, and diagonal directions, ${ }^{52}$ they are also related to quantities that appear in the computation of the Laplacian sharpness metric. For the latter they would mostly correspond to the high-pass coefficients of the Fresnelet transform.

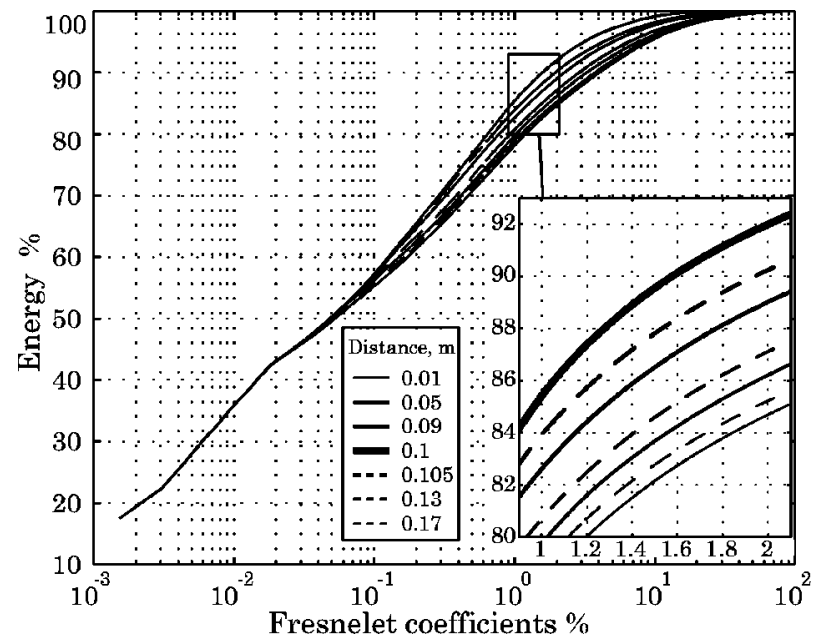

Fig. 4. Wavelet coefficient energy for several Fresnelet bases and detail (inset).

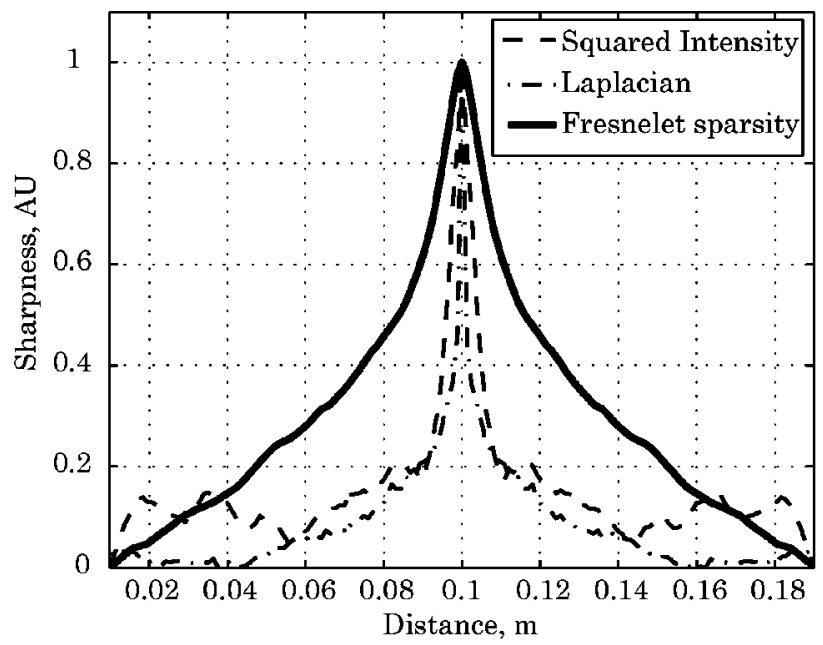

(a)
However, these are highly sensitive to noise, which makes the Laplacian sharpness metric useless in noisy conditions [see Fig. 5(b)]. Conversely, these coefficients do not contribute to our sparsity metric since they carry little energy and are removed, similarly to wavelet-based noisereduction algorithms. ${ }^{39,40}$ For this reason, our method is robust to noise, i.e., suitable for experimental situations.

In this experiment the autofocus algorithm converged to a relative precision of $10^{-3}$ in as few as nine iterations.

\section{B. Experimental Measurements}

We tested our autofocusing algorithm on experimental digital-holography data. The (unfocused) amplitude and phase in the camera plane were computed from the holograms by use of a phase-retrieval algorithm presented elsewhere. ${ }^{10}$ We then applied our autofocus algorithm to determine the focusing distance.

In the left column of Fig. 6 we show the amplitude of the unfocused wave fields for three different samples recorded in similar conditions. ${ }^{8}$ In the center column we present the reconstructed amplitude that is properly focused by use of the distance returned by the autofocus.

To better understand how the Fresnelet metric behaves compared with other metrics, we computed $S(d)$ over an extended range of distances along with the Laplacianbased and intensity-based metrics [Eq. (14)]. The corresponding curves are shown in the right column of Fig. 6 . We can see that our proposed sparsity metric behaves well even in experimental conditions. Conversely, the two other metrics are unreliable since, at best they exhibit only a local maximum for the proper distance. As a consequence any maximization procedure inevitably gets trapped in one of these false maxima.

Although the $S_{L}$ and $S_{4}$ metrics may be used successfully in incoherent imaging systems-where the defocus aberration has an effect comparable to blurring or local averaging - they are inappropriate in our case: Images with higher contrast and possibly sharper or enhanced edges may be obtained away from proper focus as a result of constructive and destructive interferences produced by

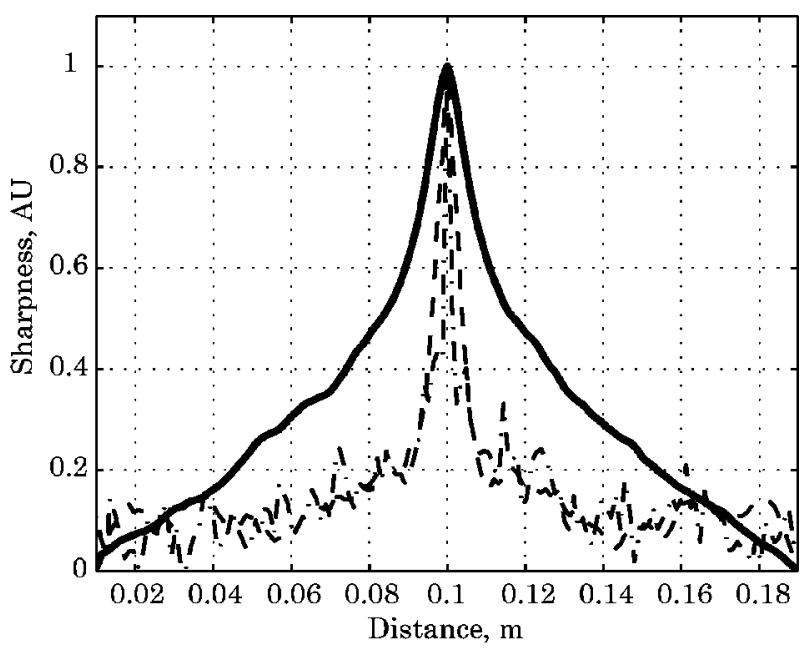

(b)

Fig. 5. Sharpness metrics as a function of distance. (a) Ideal noiseless data. (b) Noise-corrupted data: 10\% Gaussian random noise was added to the complex-valued propagated wave of Fig. 3(a). The sparsity of the Fresnelet transform is given by the energy of $\alpha$ $=1 \%$ Fresnelet coefficients. 

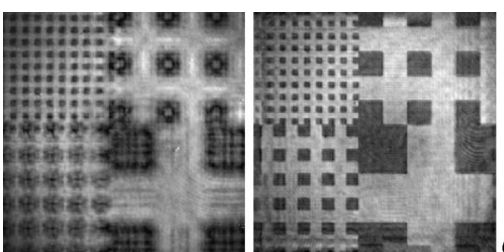

(a)
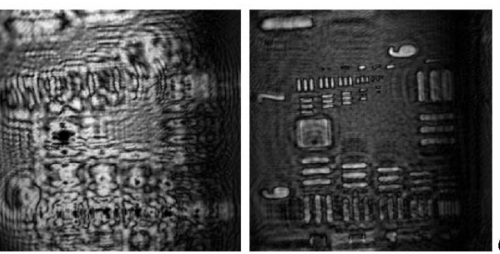

(b)
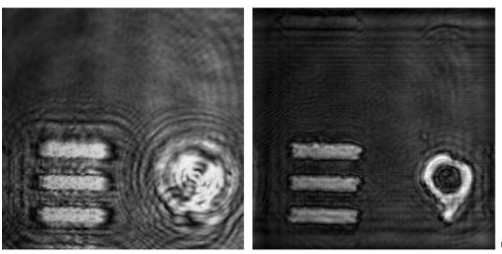

(c)

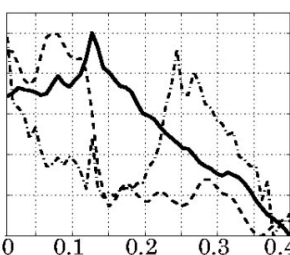

Distance, $\mathrm{m}$

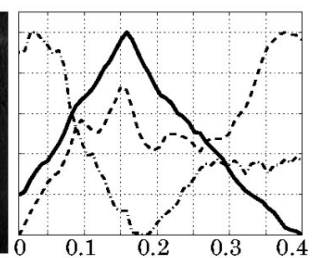

Distance, $\mathrm{m}$

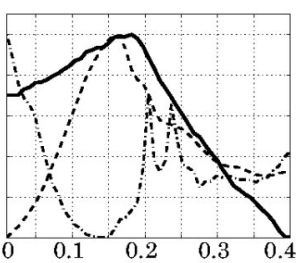

Distance, $\mathrm{m}$
Fig. 6. Out of focus (left column) and focused (center column) modulus of wave front, and sharpness metrics (right column) [Laplacian of intensity (dashed-dotted curve), squared intensity (dashed curve), and Fresnelet sparsity (solid curve)]. The experimental data were kindly provided by E. Cuche and Ch. Depeursinge, École Polytechnique Fédérale de Lausanne, Switzerland. Movies that supplement this figure are available at http:// bigwww.epfl.ch/liebling/suppinfo/A9229/index.html.

the diffraction of coherent waves. This effect is more noticeable for objects that have sharp features, as it depends on the local spatial frequencies. It also explains why the resulting curves in Fig. 6 are very different from each other despite the similarity of experimental conditions.

Since the proposed $S(d)$ metric is intrinsically based on the (coherent) reconstruction model, it avoids the shortcomings of other metrics. However, local maxima may still occur, as can be seen in Fig. 6(a): The wave diffracted by this regular pattern generates sparse representation at two different distances. A priori knowledge of the initial distance range is usually sufficient to overcome such situations. If such information is lacking, one may also find local extrema starting from varying initial distances (possibly chosen randomly) and then picking the highest of these, or one may perturb a local maximum by rerunning the maximization procedure starting at a finite-distance step away from it and then checking to see whether the result remains the same.

\section{CONCLUSION}

We have proposed a novel sharpness metric for the reconstruction of digital holograms based on the computation of the sparsity of a Fresnelet decomposition. The global maximum of this functional corresponds to a sharp reconstruction. Because it is smooth and unimodal even in the presence of noise, the localization of its maximum may be carried out without an exhaustive search but only by evaluating it for a limited number of distances. This is a crucial aspect of our metric since the cost of computing the wave front at many distances can become important. Other simple metrics that exhibit local maxima are useless because finding the global maximum requires exhaustive search. The sharpness measure has all the characteristics of a good functional; in particular, it is robust and does not require a large computational overhead since it is tightly related to our reconstruction technique. Although a wide variety of wavelets (Daubechies, etc.) may be considered for building Fresnelets, we advise the use of cubic Battle-Lemarié wavelets, since these may be constructed using B-splines, which have closed-form expressions in both time and frequency and yield a simple implementation. It is important, however, that the wavelet basis be orthonormal to ensure the validity of Eq. (13).

Finally we have subjected our technique to both synthetic (simulated) and experimental data and observed that it is suitable for real applications.

\section{ACKNOWLEDGMENTS}

The authors thank E. Cuche and Ch. Depeursinge, École Polytechnique Fédérale de Lausanne, Switzerland, for providing the experimental holograms. We acknowledge the support of the Swiss National Science Foundation (SNF). Part of this work was completed while M. Liebling was with the Biomedical Imaging Group, École Polytechnique Fédérale de Lausanne. He is currently supported by SNF fellowship PBEL2-104418.

Corresponding author M. Liebling's e-mail address is liebling@caltech.edu.

\section{REFERENCES}

1. D. Gabor, "A new microscopic principle," Nature 161, 777778 (1948).

2. J. W. Goodman and R. W. Lawrence, "Digital image formation from electronically detected holograms," Appl. Phys. Lett. 11, 77-79 (1967).

3. M. A. Kronrod, N. S. Merzlyakov, and L. P. Yaroslavskii, "Reconstruction of a hologram with a computer," Sov. Phys. Tech. Phys. 17, 333-334 (1972).

4. L. P. Yaroslavskii and N. S. Merzlyakov, Methods of Digital Holography (Consultants Bureau, New York, 1980).

5. Th. Kreis, "Digital holographic interference-phase measurement using the Fourier-transform method," J. Opt. Soc. Am. A 3, 847-855 (1986).

6. U. Schnars and W. Jüptner, "Direct recording of holograms by a CCD target and numerical reconstruction," Appl. Opt. 33, 179-181 (1994).

7. E. Cuche, F. Bevilacqua, and Ch. Depeursinge, "Digital holography for quantitative phase-contrast imaging," Opt. Lett. 24, 291-293 (1999).

8. E. Cuche, P. Marquet, and Ch. Depeursinge, "Simultaneous amplitude-contrast and quantitative phase-contrast microscopy by numerical reconstruction of Fresnel off-axis holograms," Appl. Opt. 38, 6994-7001 (1999).

9. J. W. Goodman, Introduction to Fourier Optics, 2nd ed. (McGraw-Hill, New York, 1996).

10. M. Liebling, Th. Blu, and M. Unser, "Complex-wave retrieval from a single off-axis hologram," J. Opt. Soc. Am. A 21, 367-377 (2004).

11. M. Liebling, Th. Blu, and M. Unser, "Fresnelets: new multiresolution wavelet bases for digital holography," IEEE Trans. Image Process. 12, 29-43 (2003).

12. R. A. Jarvis, "Focus optimization criteria for computer image-processing," Microscope 24, 163-180 (1976). 
13. A. Erteza, "Sharpness index and its application to focus control," Appl. Opt. 15, 877-881 (1976).

14. S. J. Erasmus and K. C. A. Smith, "An automatic focusing and astigmatism correction system for the SEM and CTEM,” J. Microsc. 127, 185-199 (1982).

15. H. Lichte, "Optimum focus for taking electron holograms," Ultramicroscopy 38, 13-22 (1991).

16. R. Yin, P. J. Flynn, and S. L. Broschat, "Position-dependent defocus processing for acoustic holography images," Int. J. Imaging Syst. Technol. 12, 101-111 (2002).

17. R. A. Muller and A. Buffington, "Real-time correction of atmospherically degraded telescope images through image sharpening," J. Opt. Soc. Am. 64, 1200-1210 (1974).

18. R. T. Brigantic, M. C. Roggemann, K. W. Bauer, and B. M. Welsh, "Image-quality metrics for characterizing adaptive optics system performance," Appl. Opt. 36, 6583-6593 (1997).

19. E. W. Justh, M. A. Vorontsov, G. W. Carhart, L. A. Beresnev, and P. S. Krishnaprasad, "Adaptive optics with advanced phase-contrast techniques. II. High-resolution wavefront control," J. Opt. Soc. Am. A 18, 1300-1311 (2001).

20. E. H. Linfoot, "Transmission factors and optical design," J. Opt. Soc. Am. 46, 740-752 (1956)

21. B. Horn, "Project MAC: Focusing," MIT Artificial Intelligence Memo 160, Massachusetts Institute of Technology, Cambridge, Mass. (1968), ftp://publications.ai.mit.edu/aipublications/pdf/AIM-160.pdf.

22. P. Grossman, "Depth from focus," Pattern Recogn. Lett. 5, 63-69 (1987)

23. A. W. Lohmann, D. Mendlovic, and Z. Zalevsky, "Digital method for measuring the focus error," Appl. Opt. 36, 72047209 (1997).

24. J. R. Fienup, "Synthetic-aperture radar autofocus by maximizing sharpness," Opt. Lett. 25, 221-223 (2000).

25. J. R. Fienup and J. J. Miller, "Aberration correction by maximizing generalized sharpness metrics," J. Opt. Soc. Am. A 20, 609-620 (2003)

26. J. Gillespie and R. A. King, "The use of self-entropy as a focus measure in digital holography," Pattern Recogn. Lett. 9, 19-25 (1989).

27. L. Sica, "Image-sharpness criterion for space-variant imaging," J. Opt. Soc. Am. 71, 1172-1175 (1981).

28. J. R. Fienup, "Invariant error metrics for image reconstruction," Appl. Opt. 36, 8352-8357 (1997)

29. F. C. A. Groen, I. T. Young, and G. Ligthart, "A comparison of different focus functions for use in autofocus algorithms," Cytometry 6, 81-91 (1985).

30. P. Ferraro, G. Coppola, S. De Nicola, A. Finizio, and G. Pierattini, "Digital holographic microscope with automatic focus tracking by detecting sample displacement in real time," Opt. Lett. 28, 1257-1259 (2003).

31. P. R. Hobson and J. Watson, "The principles and practice of holographic recording of plankton," J. Opt. A Pure Appl. Opt. 4, 34-49 (2002).

32. J. Widjaja and S. Jutamulia, "Use of wavelet analysis for improving autofocusing capability," Opt. Commun. 151, 12-14 (1998).

33. F. Rooms, A. Pizurica, and W. Philips, "Estimating image blur in the wavelet domain," in Proceedings of the Fifth Asian Conference on Computer Vision (ACCV) [Asian Federation of Computer Vision Societies (AFCV), Victoria, Australia, 2002], pp. 210-215.

34. L. Onural and M. Kocatepe, "Family of scaling chirp functions, diffraction, and holography," IEEE Trans. Image Process. 43, 1568-1578 (1995).

35. S. G. Mallat, "A theory for multiresolution signal decomposition-the wavelet representation," IEEE Trans. Pattern Anal. Mach. Intell. 11, 674-693 (1989).

36. B. A. Olshausen and D. J. Field, "Emergence of simple-cell receptive field properties by learning a sparse code for natural images," Nature 381, 607-609 (1996).

37. D. J. Field, "Relations between the statistics of natural images and the response properties of cortical cells," J. Opt. Soc. Am. A 4, 2379-2394 (1987).

38. D. L. Donoho, "Superresolution via sparsity constraints," SIAM (Soc. Ind. Appl. Math.) J. Math. Anal. 23, 1309-1331 (1992).

39. J. B. Weaver, X. Yansun, D. M. Healy, Jr., and L. D. Cromwell, "Filtering noise from images with wavelet transforms," Magn. Reson. Med. 21, 288-295 (1991).

40. D. L. Donoho and I. M. Johnstone, "Ideal spatial adaptation by wavelet shrinkage," Biometrika 81, 425-455 (1994).

41. D. L. Donoho, M. Vetterli, R. A. DeVore, and I. Daubechies, "Data compression and harmonic analysis," IEEE Trans. Inf. Theory 44, 2435-2476 (1998).

42. JPEG 2000 Image Coding System, ISO/IEC FCD154441:2000 [International Organization for Standardization (ISO), Geneva, Switzerland, 2000], http://www.iso.org/.

43. M. Zibulevsky and Y. Y. Zeevi, "Extraction of a source from multichannel data using sparse decomposition," Neurocomputing 49, 163-173 (2002).

44. S. G. Mallat, A Wavelet Tour of Signal Processing (Academic, San Diego, Calif., 1998).

45. G. Battle, "A block spin construction of ondelettes. 1. Lemarié functions," Commun. Math. Phys. 110, 601-615 (1987).

46. P. G. Lemarié-Rieusset, "Ondelettes à localisation exponentielle," J. Math. Pures Appl. 67, 227-236 (1988).

47. Matlab Function Reference, Vol. 2: F-O (The MathWorks, Natick, Mass., 2002).

48. G. E. Forsythe, M. A. Malcolm, and C. B. Moler, Computer Methods for Mathematical Computations (Prentice Hall, Englewood Cliffs, N.J., 1977)

49. R. P. Brent, Algorithms for Minimization without Derivatives (Prentice Hall, Englewood Cliffs, N.J., 1973).

50. W. H. Press, S. A. Teukolsky, W. T. Vetterling, and B. P. Flannery, Numerical Recipes in $C$, 2nd ed. (Cambridge U. Press, Cambridge, UK, 1992).

51. R. Sedgewick, Algorithms, 2nd ed. (Addison-Wesley, Reading, Mass., 1988).

52. M. Unser and Th. Blu, "Wavelet theory demystified," IEEE Trans. Signal Process. 51, 470-483 (2003). 